\title{
Téoros
}

Revue de recherche en tourisme

\section{La méthode des cas : un outil pédagogique à privilégier pour l'enseignement du tourisme}

\section{Michel Archambault}

Volume 11, numéro 1, mars 1992

La formation en tourisme : à la croisée des chemins

URI : https://id.erudit.org/iderudit/1078943ar

DOI : https://doi.org/10.7202/1078943ar

Aller au sommaire du numéro

Éditeur(s)

Université du Québec à Montréal

ISSN

0712-8657 (imprimé)

1923-2705 (numérique)

Découvrir la revue

Citer cet article

Archambault, M. (1992). La méthode des cas : un outil pédagogique à privilégier pour l'enseignement du tourisme. Téoros, 11(1), 16-16.

https://doi.org/10.7202/1078943ar d'utilisation que vous pouvez consulter en ligne. 
Quoi de plus naturel pour enseigner le tourisme que de se servir d'exemples puisés à même la multitude d'entreprises qui oeuvrent dans cette industrie! L'opportunité d'étudier et de réfléchir à partir du vécu constitue un avantage pour bien préparer la releve de demain. Aussi est-il imperieux, dirons certains, d'encourager le recours à la méthode des cas pour inculquer un sens pratique à nos enseignements et confronter notre population étudiante avec $c e$ qui se passe dans la «vraie view.

La méthode des cas se présente comme un outil pédagogique puissant qui favorise le lien entre la pratique et la thérie, entre l'université et le monde du travail. Un bref rappel historique: implantée d'abord à la faculté de droit de l'Université de Harvard, la méthode des cas s'est rapidement rêpandue dans l'enseignement de diverses disciplines à caractère professionnel comme la gestion et la médecine. Elle est aujourd'hui utilisée par plusieurs grandes universités américaines et en particulier par les «Business Schoolss.

La démarche pédagogique s'appuie sur l'illustration d'une situation concrète où on demande aux étudiants de trouver une solution ou d'esquisser un scénario d'actions et de décisions ăprendre pour résoudre le ou les problèmes présentés. Certes il s'avère intéressant de partir de problèmes rencontrés pour dégager par la suite une théorie donnée. L'apprentissage du point de vue du transfert des connaissances s'en trouve facilite car l'application de la solution est immédiate.

La rédaction d'un cas exige une bonne collecte d'informations de l'entreprise étudiée et du secteur auquel elle appartient. Ordinairement, une série d'entretiens avec les dirigeants situe davantage les fonctions essentielles de l'organisation en relation avec l'objet d'étude. Le rédacteur du cas pourra consacrer plus de 100 heures à colliger l'information, degager les points saillants et présenter le tout dans une forme littéraire vivante. A titre d'exemple, pour le cas Mont St-Sauveur International, j'ai eu l'occasion

\footnotetext{
Monsieur Michel Archambault est directeur de la Chaire en tourisme et professeur au département d'études urbaines et touristiques a l'Université du Québec à Montréal.
}

de diriger, avec un collè gue, deux rédacteurs (un généraliste et un spécialiste en finance) qui ont passé environ chacun 75 heures pour compléter unepremière version du cas. Nous avons tenu trois rencontres avec les principaux dirigeants afin de peaufiner l'information recueillie. Rappelons que le cas portait sur les choix stratégiques de $1^{\dagger}$ entreprise et sur sa capacité financière à assurer son dêveloppement.

La méthode des cas implique une participation active à la discussion; elle appelle un jugement des faits présentés; elle favorise l'application de certains outilsd'analyse pour évaluer l'impact des choix élaborés; elle demande aux étudiants un sens du réalisme: trouver une solution applicable compte tenu des contraintes intemes et externes. Comme chaque situation est unique en soi, $l^{\prime}$ 'étudiant doit justifier ses recommandations selon son analyse des faits présentés et sur la base souvent d'hypothèses réalistes. Sa capacité de créativité et d'innovation en termes de solutions est donc mise à contribution. En effet, plusieurs «bonnes» réponses peuvent résoudre un problème.

Enseigner par la méthode des cas exige une bonne préparation (lecture du cas et analyse préalable), un local où la disposition physique des pupitres et $l^{\prime}$ acoustique encouragent les échanges, un climat de complicité entre les participants. Cetteméthode pédagogique axée sur l'analyse et l'action n'exclut pas le recours à des lectures dirigées qui viennent compléter l'apprentissage tout en seccurisant l'étudiant dans la préparation du cas.

L'enseignement des disciplines dites professionnelles s'inspire de plus en plus de l'environnementdanslequelellesévoluent. Référer à un exemple précis pour illustrer un concept ou une théorie permet de mieux ancrer nos enseignements. Le tourisme $s^{4}$ avère une discipline où on peut s'appuyer sur de nombreux exemples pour rédiger des cas. Pensons, à titre d'illustration, à la stratégie de mise en marche d'un site touristique pour présenter certaines théories du marketing; ou à un programme de formation du personnel d'un établissement hôtelier pour étudier les principes et l'importance de l'accueil; ouaux contraintes et aux étapes nécessaires dans l'établissement et l'aménagement d'un centre de villégiature pour faire ressortir les liens entre le législatif, le politique et les impératifs d'une telle implantation.
Nous pouvons également citer l'impact de la technologie sur les réseaux de distribution et les systèmes de réservations par l'étude d'un voyagiste spécifique; ou analyser les modifications de comportements de la clientèle et ses effets sur la viabilité d'une entreprise; ou les relations entre l'expansion et les structures d'aco's aux capitaux. Cette liste non exhaustive suppose évidemment la collaboration des entreprises concemées. Par leur participation, ces demières manifestent une ouverture d'esprit et contribuent par le fait même à rehausser la qualité du processus d'apprentissage. C'est également une occasion de tisser des liens étroits entre l'université et l'entreprise.

Malgré le nombre élevé d'entreprises touristiques, l'utilisation de cas dans l'enseignement du tourisme demeure restreint. J'ai eu I'opportunité de diriger quelque sept a huit cas dans ce secteur. Harvard en a produit également quelques-uns. Il y a certes place pour l'addition de plusieurs cas dans nos programmes de formationetce, pour le bénéfice des professeurs, des étudiants et des entreprises. Les quelques exemples mentionnés ci-haut illustrent la complexité et la diversité de l'industrie touristique.

En projetant les étudiants dans une situation réelle, on favorise la connaissance du domaine étudié. En leur demandant de prendre position face à des problèmes concrets, on les incite à développer leur capacité d'analyse et d'argumentation des faits. Par ailleurs, la méthode des cas requiert des qualités d'animation et de direction de groupe pour stimuler la participation des étudiants. La discussion doit s'orienter vers l'action alimentée par le bon usage des questions du professeur. Ce demier $s^{*}$ assure que le groupe structure l'information présentée de façon pêle-mẻle et traduit en actions les suggestions émises. Enfin, la méthode des cas implique et encourage des relations suivies avec ce qui se passe sur le terrain pour renouveler et enrichir la matière grise!

J'invite donc le lecteur à me souligner son intérêt pour la création de nouveaux cas en tourisme ou porter à ma connaissance l'existence de cas tirés de cette industrie. Nous pourrionsainsiéventuellementconstituer une banque de cas utile pour tous. 